\title{
Conical intersection dynamics of pyrimidine nucleosides tracked with sub-20-fs UV pulses
}

\author{
Rocio Borrego-Varillas ${ }^{1}$, Artur Nenov ${ }^{2}$, Lucia Ganzer ${ }^{1}$, Aurelio Oriana ${ }^{1}$, Irene Conti $^{2}$, Ines \\ Delfino $^{3}$, Cristian Manzoni ${ }^{1}$, Marco Garavelli ${ }^{2}$ and Giulio Cerullo ${ }^{1,1}$ \\ ${ }^{1}$ IFN-CNR, Dipartimento di Fisica, Politecnico di Milano, Piazza Leonardo da Vinci 32, I-20133 \\ Milano, Italy \\ ${ }^{2}$ Dipartimento di Chimica Industriale, Università degli Studi di Bologna, Viale del Risorgimento 4, I- \\ 40136 Bologna, Italy \\ ${ }^{3}$ Dipartimento di Scienze Ecologiche e Biologiche, Università della Tuscia, Largo dell’Università snc, \\ I-01100 Viterbo, Italy
}

\begin{abstract}
By combining transient absorption spectroscopy with sub-20-fs UV pulses and $a b$ initio numerical simulations we follow the ultrafast dynamics in pyrimidine nucleosides and visualize the passage through conical intersections presiding excited state deactivation.
\end{abstract}

\section{Introduction}

When ultraviolet (UV) radiation is absorbed by DNA, the electronic energy acquired by the molecule is efficiently converted into vibrational energy on an ultrafast timescale, preventing photochemical reactions which might induce mutations or strand breaks [1]. In those ultrafast passages, conical intersections (CIs) play a crucial role and have been proposed as responsible for sub-picosecond fluorescence lifetimes in nucleobases [2]. Over the past years many studies have been performed to shed light in the fate of photoexcitations in DNA, although most of them with limited (>100 fs) temporal resolution $[3,4]$. In this work we combine transient absorption (TA) spectroscopy using sub-20 fs UV pump pulses and broad UV spectral coverage with hybrid quantum mechanics /molecular mechanics (QM/MM) computations to study the ultrafast relaxation dynamics of pyrimidine nucleosides (uridine and 5-methyluridine).

\section{Methods}

Ultrafast TA measurements were carried out on a home-made pump-probe setup, based on a Ti:Sapphire laser generating 100-fs pulses at $800 \mathrm{~nm}$ wavelength and $1 \mathrm{kHz}$ repetition rate. By second harmonic generation of a broadband visible noncollinear OPA (NOPA) followed by compression with a prism pair, we generate sub-20-fs UV pulses in the 260$280 \mathrm{~nm}$ range [5], which we fully characterize by two-dimensional spectral interferometry [6]. A broadband probe (covering $270-700 \mathrm{~nm}$ ) is obtained through white light continuum generation of either the fundamental or its second harmonic in a calcium fluoride plate. 
Nucleosides samples were prepared in a water-based buffer solution and measured in a laminar jet flow configuration. The QM/MM scheme couples a state-of-the-art ab-initio multireference dynamically correlated description (CASPT2) of the bare nucleobase with an explicit classical atomistic model (AMBER force field) of the sugar and the solvent [7]. A topological analysis of the populated excited states (ES) is supplemented by mixedquantum classical molecular dynamics simulations realised through an ensemble of 60 room temperature trajectories initiated in the lowest bright $\pi \pi^{*}$ state and employing Tully's fewest switches surface hopping algorithm to follow the ES deactivation during the first $500 \mathrm{fs}$ (uridine) / $1000 \mathrm{fs}$ (5-methyluridine) after photoexciation. The level of theory employed was SS-CASPT2/SA-2-CASSCF(10,8)/ANO-L[3s2p1d/2s1p] comprising all valence $\pi$-orbitals.

\section{Results and Discussion}

Figure 1 shows TA measurements on uridine and 5-methyluridine in the UV range following excitation at $270 \mathrm{~nm}$. For both nucleosides we observe for the first time a stimulated emission (SE) band, extending from 300 to $360 \mathrm{~nm}$ and assigned to the photoexcited bright $\pi \pi^{*}$ state. In both cases the SE band shows a very fast decay, fitted with a time constant of $\sim 95 \mathrm{fs}$, which is tentatively associated to ES deactivation through a CI. The SE band gives way to a photoinduced absorption (PA) band, which we assign to a hot ground state (GS), red-shifted with respect to the GS absorption; this band in turn decays on the picosecond timescale due to vibrational cooling in the GS. In 5-methyluridine the SE band shows a bi-exponential decay with a slower tail featuring a 2-ps time constant, suggesting the involvement of a slower deactivation pathway of the $\pi \pi^{*}$ state.
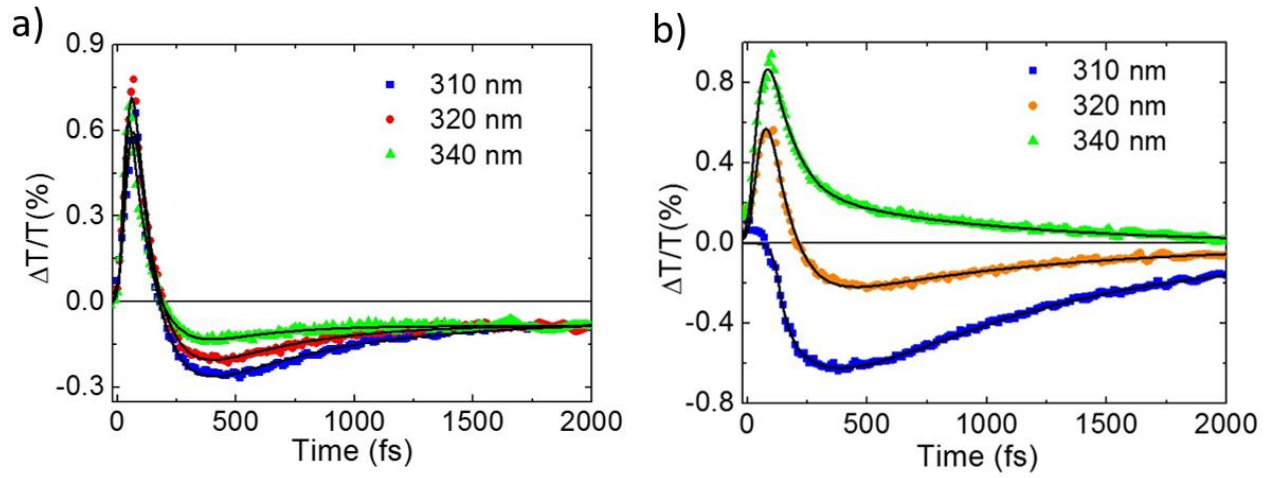

Fig. 1. (a) Uridine and (b) 5-Methyluridine TA dynamics at different probe wavelengths, experimental data in symbols and fits in solid lines.

We recently identified, through static computations within the hybrid QM(CASPT2)/MM(AMBER) framework two deactivation channels from the lowest bright $\pi \pi *$ state, initially populated by $270 \mathrm{~nm}$ UV light, to the GS, involving ring-puckering and ring-opening [8]. Both deactivation pathways are associated with CIs reached over small barriers $(0.1-0.2 \mathrm{eV})$ making them potentially responsible for the ballistic coherent $<100 \mathrm{fs}$ decays observed experimentally. By performing mixed quantum-classical dynamics simulations on room temperature samples of uridine and 5-methyluridine we addressed the involvement of the two proposed routes in the deactivation mechanism, as well as the lifetimes associated with the $\pi \pi^{*} \rightarrow$ GS decay. Representative trajectories for uridine a 5methyluridine are shown in Figures 2(a) and 2(b), respectively. In particular, we identify the ring puckering as the exclusive ES deactivation mechanism in uridine when pumping is 
performed at $270 \mathrm{~nm}$. The ring deformation is responsible for the sub-100 fs decrease of the $\pi \pi^{*} \rightarrow$ GS energy gap and, consequently, for the disappearance of the SE.

Instead, 5-methyluridine is found to decay on a ps time scale, i.e. an order of magnitude slower than uridine, with no hopping events observed prior to $200 \mathrm{fs}$. Again, ring-puckering is found to be the dominant deactivation channel, with a few trajectories exploring an oxygen-out-of-plane deactivation route. According to our simulations, it is not the ultrafast sub-100 fs component of the decay of the SE band, observed in the transient spectra (Figure 1d), that is to be associated with the decay to the GS but rather the 2-ps component. In the light of these findings the sub-100 fs component could be associated with: a) wavepacket departure from the FC region and loss of coherence; and/or b) population transfer to the $n \pi^{*}$ state.
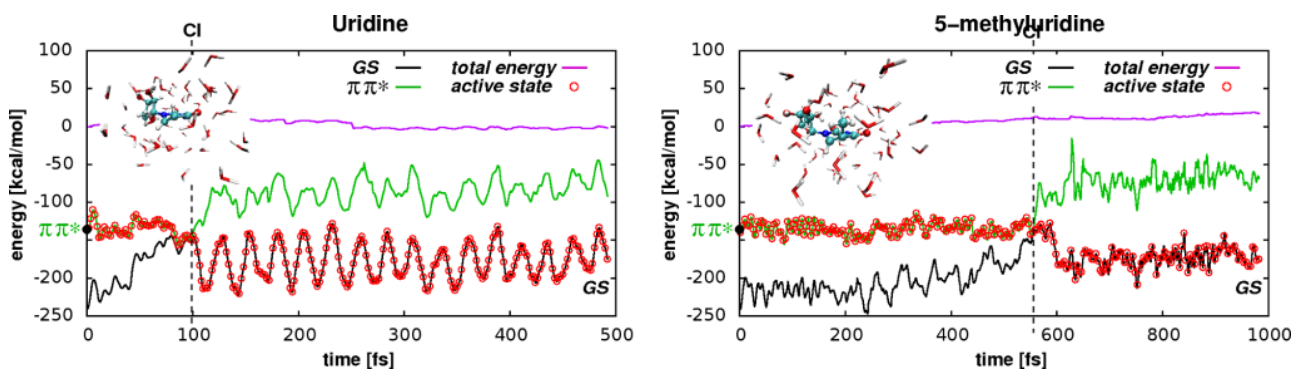

Fig. 2. Representative trajectories for uridine (a) and 5-methyluridine (b) at the QM(SS-CASPT2/SA2-CASSCF $(10,8)) / M M(A M B E R)$ level of theory, showing the decay from the initially populated $\pi \pi^{*}$ state (red) to the ground state (black) through a puckered conical intersection.

\section{References}

[1] D. L. Miller and M. A. Weinstock J. Am. Acad. Dermatol. 30, 774 (1994).

[2] J.-M. L. Pecourt, J. Peon and B. Kohler J. Am. Chem. Soc. 122, 9348 (2000).

[3] C. E. Crespo-Hernández, B. Cohen, P. M. Hare and B. Kohler Chemical reviews 104, 1977, (2004).

[4] C. T. Middleton, K. de la Harpe, C. Su, Y. K. Law, C. E. Crespo-Hernández, B. Kohler Annu. Rev. Phys. Chem. 60, 217 (2009).

[5] R. Borrego-Varillas, L. Ganzer, G. Cerullo and C. Manzoni, Appl. Sci. 8, 2076 (2018).

[6] R. Borrego-Varillas, A. Oriana, F. Branchi, S. De Silvestri, G. Cerullo and C. Manzoni, J. Opt. Soc. Am. B 32, 1851 (2015).

[7] O. Weingart, A. Nenov, P. Altoé, I. Rivalta, J. Segarra-Martí, I. Dokukina and M. Garavelli, J. Mol. Model., in press (2018) DOI: 10.1007/s00894-018-3769-6.

[8] A. J. Pepino, J. Segarra-Martí, A. Nenov, R. Improta and M. Garavelli, J. Phys. Chem. Lett. 8, 1777 (2017). 